\title{
Evaluation and Application of MIDAS v2.0
}

\author{
Sandra G. Hart \\ NASA-Ames Research Center \\ Moffett Field, CA \\ David Dahn \\ Micro Analysis \& Design Inc. \\ Boulder, CO.
}

Adolph Atencio

US Army Aeroflight Dynamics Directorate (Ret) NASA-Ames Research Center Moffett Field, CA

K. Michael Dalal

Raytheon STX Corporation Moffett Field, CA

Copyright (C) 2001 Society of Automotive Engineers, Inc.

\begin{abstract}
Version 2.0 of the Man-Machine Design and Analysis System (MIDAS) was released in 2001. It provides tools to describe an operating environment, mission, and equipment. User-defined goals, procedures, and knowledge interact with and are modified by models of perception, memory, situation awareness, and attention and constrained by the environment. Output of simulations that demonstrate or evaluate new capabilities or answer questions posed by customers are presented graphically and visually. MIDAS has been used to model different professions (soldiers in protective gear, air traffic controllers, astronauts, nuclear power plant operators, pilots), missions (e.g., flying, target designation, underwater exploration, police dispatch) and environments (e.g., battlefields, civil airspace, ocean floor, control rooms, low earth orbit). A recent independent evaluation of MIDAS V2.0 is reviewed.
\end{abstract}

\section{INTRODUCTION}

Development of the Man-Machine Integration Design and Analysis System (MIDAS) began in 1983 as a joint Army/NASA exploratory development effort. The Program was primarily funded by the US Army Aeroflightdynamics Directorate and led by US Army scientists and engineers. E. James Hartzell led the program until 1995, followed by Barry Lakinsmith and R. Jay Shively. In 2001, responsibility was transferred to
NASA's Human Factors Research and Technology Division.

The primary goal was to develop an engineering environment that contained the tools and models needed by crew station developers during the conceptual design phase. It would enable rapid prototyping and offer early integration and visualization of developing designs to foster communication among members of a design team. A secondary goal was to advance the capabilities and use of computational representations of human performance in design. As an early and ambitious pioneer, MIDAS has clearly achieved this objective. In addition, it was hoped that MIDAS would serve as a framework for integrating useful research results and models. A final goal was to transfer technologies to the larger community of industry, government, and university researchers and designers. This process continues under the auspices of Army and NASA "commercialization" agreements.

It has been estimated that as much as $80 \%$ of the lifecycle cost of developing an aircraft is determined in the conceptual design phase; after hardware is built, it is difficult to correct errors or modify the design. MIDAS was conceived as a method of offering designers an opportunity to "see" their design being operated by a human model in a virtual environment and to ask "what if" questions about the impact of design alternatives and candidate procedures early in design (1) to prevent costly retrofits and inefficient training "band-aids" for clumsy designs. All too often in the past, human operators have had to compensate for poorly integrated subsystems and periods of high workload or stress. 
Initial interest in computational human performance modeling tools, such as MIDAS, was prompted by a desire to make objective decisions about which tasks to automate and minimum crew size for Army helicopters, commercial airliners, and Army tanks. These interests are what prompted early development of "resource" models and "workload" prediction capabilities, as operator workload was thought to be a limiting factor in system performance.

MIDAS has progressed through eight development phases, each culminating in demonstration of new capabilities. The initial architecture was built in a skeletal form with the expectation that it would be populated with new models, information and tools. Initially, MIDAS consisted of a number of integrated workstations that contributed simulations of the mission, operator, and environment to the designer's workstation. Models of human performance and behavior were used to evaluate different aspects of the task or equipment design statically or dynamically. The component models and tools were coordinated by a discrete, scaled-time simulation. Unlike man-in-the-loop simulations, there was no requirement for operating in real time. The results of an analysis were presented graphically and/or visually in the form of a virtual simulation of a "manned" mission. One form of MIDAS output supports scenarioindependent analysis of crew station layout (e.g., visibility, legibility, reach) and is compatible with military standards. The other provides a dynamic simulation that can be visualized from different perspectives, with graphic activity traces, task load timelines, information requirements, and mission performance measures (2). Although the number of computers supporting MIDAS have decreased as computational speed and capability have increased, MIDAS is still hosted on a Silicon Graphics Workstation, although $\mathrm{C}++$ has replaced Common Lisp and FORTRAN.

As MIDAS matured, it offered an increasing number of tools for modelers at Ames and Beta test sites. It has been used to model different types of operators (e.g., US Army personnel wearing protective gear, air traffic controllers, astronauts, nuclear power plant operators, helicopter and airline pilots, and 911 dispatchers), missions (e.g., flying, target designation, underwater exploration, police dispatch), and environments (e.g., nap-of-the earth helicopter flight, civilian airspace, ocean floor, control room, low-earth orbit). Such simulations, some of which will be described below, were developed to demonstrate or evaluate new capabilities or to address questions posed by customers from NASA, US Army, US Navy, the Department of Energy, and the Richmond, CA Police Department.

The primary challenge faced by MIDAS developers then (and now), is the gap between what is known about human behavior from psychological models and laboratory research and the types of information and models needed to design and evaluate new procedures or a new system. The precise numerical predictions and neat algorithms that would allow designers to incorporate human factors considerations into developing systems did not (and still do not) exist. To some extent this is because humans are enormously complex, adaptive, and diverse. To a greater extent, however, it reflects many years of reliance on the time-honored practice of basing design decisions on judgment calls made by experienced engineers or test pilots. The great flaw of this approach is that relying on "lessons learned" from past successes or failures will doom designers to solving problems that have already happened but failing to anticipate problems that have not yet occurred. A more objective and comprehensive assessment of design questions through computational design tools offers the possibility of discovering potential problems or interactions that have not yet been encountered before.

Given the apparent dearth of models of human performance and behavior needed to populate MIDAS, the National Research Council Committee on Human Factors was approached in 1987 to offer guidance to the program. Following two years of deliberation and interaction with members of the NASA/Army Program, the 12-member panel published their report (3). It offered a number of recommendations and suggested that progress was feasible in the areas of mission analysis, workload, visual scanning, detectablity, and legibility, topics that did, in fact, form an early focus for MIDAS. Other challenges involved integration among component models and tools, especially those associated with variations in granularity, precision and temporal resolution. Although no longer operating at the cutting edge of computer science, MIDAS taxed the capabilities of a room-full of computers in its early years.

MIDAS was developed "in-house" by Army and NASA researchers and support service contractors. For example, Ms. Ranuka Shankar developed a scheduling model (4). Differences in the salience of stimuli is represented by a model of early attention, patterned after the work of Remington and Johnston (5). Mr. R. Jay Shively and his colleagues developed a situation awareness (SA) model (6). Extramural relationships with universities and private and government research organizations offered models, research results, and independent evaluation of component models. Development of a 3D, dynamic anthropometric model to represent the human operator was an early and critical requirement. A grant to Dr. Norman Badler at the University of Pennsylvania (7) initiated the development of a realistic mannequin, Jack, that can be scaled from the $5^{\text {th }}$ percentile woman to the $95^{\text {th }}$ percentile man and placed within a $3 \mathrm{D}$ virtual workstation that can be viewed from the perspective of Jack's eyes. A model of binocular vision developed by Dr. Aries Arditi from the Lighthouse of New York was fully integrated with Jack's head position and point of regard and with a visibility model developed by Drs. James Bergen and Jeff Lubin from the SRI/David Sarnoff Research Center (8). A model of display layout principles was developed by Dr. Christopher Wickens at the University of Illinois (9). The task loading model, based on Wicken's Multiple Resource Model principles, was patterned after the approach developed by Mr. Theodore Aldrich (10). 
Decision making behavior is represented at several levels in MIDAS, one of which follows the Dr. Jens Rasmussen's distinction between skill-, rule-, knowledgebased behaviors (11). Other tools were developed to facilitate modeling of 3D environments, controls, displays and so on, using libraries of primitive objects and animation.

\section{MIDAS APPLICATIONS}

ARMY MISSION - The first model developed with the initial suite of MIDAS tools in 1985 was of a military mission performed by a Cobra $\mathrm{AH}-1$ helicopter. The simulated attack on a convoy occurred over a DMA database of the Fulda Gap. Visualization of the helicopter nap of the earth flight was from a "God's-eye" perspective.

UNMANNED UNDERWATER VEHICLE - During the same time period, the US Navy requested the development of a MIDAS model of a notional unmanned vehicle that might seek out and destroy mines on the ocean floor. The goal was conceptual exploration of the ideas using graphic representations of the vehicle, environment, and mission. The MIDAS model represented the ocean floor (viewed from the perspectives of the vehicle's camera and the surface ship) and the slow-moving search mission over the ocean floor.

911 DISPATCH - In 1993, a Cooperative Research and Development Agreement (CRDA) was signed with Communications Research Company (CRC) which designs and integrates high-speed wireless communication and navigation systems for emergency response vehicles and 911 dispatch stations. The Richmond police department partnered with the MIDAS team to study the procedures used in their 911 facility using a MIDAS representation of the 911 dispatch console geometry and evaluate a prototype graphical dispatch decision aid under consideration. Analysis software on a laptop computer was used to record over 75 hours of data on the frequency, duration, and types of 911 dispatch operator activity at Richmond PD. These data were used by MIDAS researchers to support several key design recommendations to CRC.

NUCLEAR POWER PLANT DESIGN - ONE of MIDAS' first applications examined advanced automation options for next-generation nuclear power plant consoles in collaboration with Westinghouse (12). The simulated mission involved diagnosis of a steam generator fault to compare performance and workload using the existing layout to one that offered an electronic checklist merged with one of the displays. The MIDAS simulation concentrated on the Senior Reactor Operator and his communications with other operators. Simulated interruptions not only delayed his "actions" but also interfered with his ability to "remember" where he had left off before the interruption. This was one of the first demonstrations of MIDAS' utility in a domain quite different than that for which it was developed and included a new class of events - - interruptions. The results demonstrated the potential value of the electronic checklist but also suggested potential problems with the requirement to page between or scroll within displays to accomplish procedures.

AIR WARRIOR - THE US Army initiated the Air Warrior program to develop and integrate an improved Mission Oriented Protective Posture (MOPP) ensemble for helicopter aircrews (Figure 1). At the time of the study, aircrew MOPP clothing and equipment was bulky, uncomfortable, restrictive, and not tailored for the needs of aircrew. There was a concern that flying using this equipment might degrade performance or flight safety. The MIDAS Air Warrior study was the most ambitious application that had been undertaken to date. It established baseline performance measurements with and without MOPP equipment (13). The AH-64D Longbow Apache cockpit was modeled in MIDAS using computer aided design files supplied by the manufacturer. The activities performed by the copilot/gunner in the front seat of the helicopter were the focus of the simulation. The co-pilot/gunner was modeled using the Jack $^{\circledR}$ human anthropometric modeling tool (7) and placed in the CAD representation of the cockpit Each simulation contained over 400 discrete activities such as display fixations, control manipulations, and crew decisions. The investigation concentrated on the co-pilot's ability to access controls and displays during a simulated battlefield scenario that involved designating targets on the battle field and

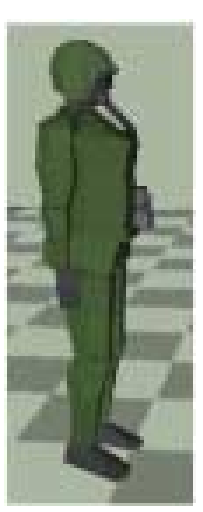

Figure 1: MOPP gear preparing for weapons launch. Performance measures included task timelines and workload imposed by an airto-ground attack scenario. Two human anthropometric sizes (by stature) were simulated to represent a $95^{\text {th }}$ percentile male and a $5^{\text {th }}$ percentile female. With the shoulder harness locked, the smallest stature body failed to reach eight of nine critical cockpit switches, even with full seat adjustment. And the vision model predicted a visual field restriction due to the MOPP protective mask, a finding supported by pilot comments. Data supporting aggregate measures of performance were collected and reported, quantifying workload, exposure time, and total mission time effects from each simulation experiment permutation.

SHORT HAUL CIVIL TILTROTOR (SHCT) - Tiltrotor aircraft combine the speed and range of a turboprop aircraft with the ability to take off and land in a vertical mode like a helicopter. These aircraft will transport passengers from city center to city center and from satellite airports to major hub airports. NASA has been evaluating a 40-passenger civil version with large bladed propellers (rotor blades) on nacelles that may be rotated from airplane mode to helicopter mode during landing. MIDAS was used to evaluate human performance issues related to crew procedures and pilot workload for a tiltrotor flying a steep, low-noise approach to a vertiport (14). The simulated scenario was of a normal approach 
interrupted by a commanded go-around at the landing decision point. The simulation contrasted the use an automated discrete nacelle control mode with a fully manual nacelle control mode for the go-around. The MIDAS simulation (Figure 2) showed that task loading was high during the approach and emergency goaround; pilot's workload was near capacity throughout. The emergency go-around in manual nacelle mode was more demanding, resulting in additional time requirements to complete tasks.

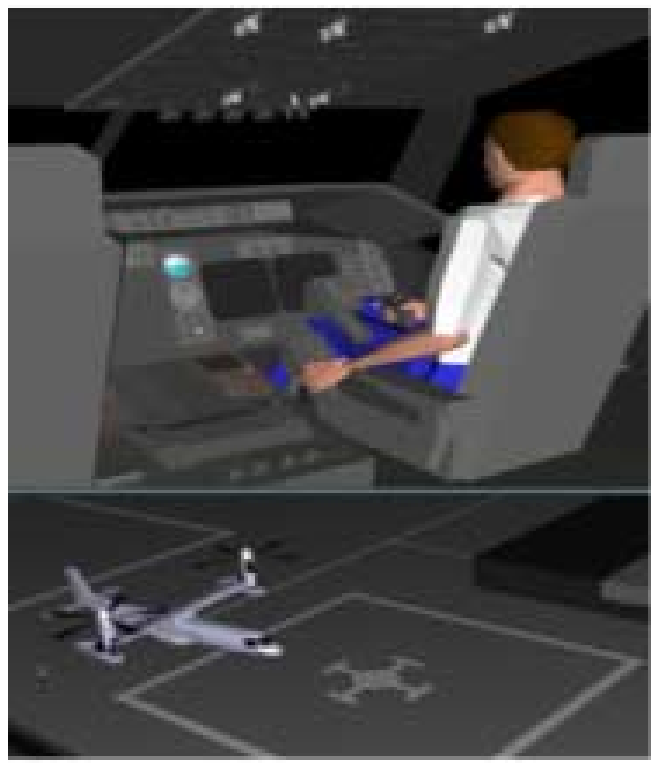

Figure 2: Civil Tiltrotor cockpit

COMMERCIAL TRANSPORT OPERATIONS - Four simulations were conducted using the "Air-MIDAS" version in support of NASA's Advanced Air Transportation Technology (AATT) Program (15). Some of the issues were studied included aircrew's processing of ATC clearance delivered via voice or data link, different flight path automation options, the relationship between clearance timing and flight-path fuel efficiency, and the influences of weather, traffic and task interruptions. Sequences and timing of crew actions were derived from pilot interviews and data from a related simulator study. Distributions for task times were created, probabilities for various forms of interruption were added, and the entire study was exercised 100 times for each fact and level combination using a Monte Carlo approach. Since the demands of processing clearances were shared between the pilot and copilot, they were treated as a composite "operator". The results demonstrated progressive decrements in performance as clearances were issued closer to the desired descent point. Crews shifted from the flight management system to a simpler form of automation earlier in the descent when receiving voice clearances. Results from this simulation were precisely those needed by terminal area automation developers to define desired clearance issuance windows. An extension of this application compared the predictions of the model with those from a piloted simulation conducted in the 747-400 simulator in Crew-Vehicle Flight Simulation Facility at Ames (16). Four human flight crews and simulated pilots in MIDAS "flew" descent profiles with the same conditions of speed, crossing restriction, and distance to top of descent. The results demonstrated MIDAS' effectiveness in predicting flight-crew performance. Additional AirMIDAS simulations have been performed to examine various implications of the "free flight" concept; constraints and requirements for controlled airspace, traffic alerting, and decision aids. Recommendations for the design of alerting systems required to maintain safe buffers around aircraft with self-separation were based on human performance constraints identified in MIDAS simulations. To meet the unique demands of modeling this environment, Air MIDAS is operated without the visualization capabilities of MIDAS, and has incorporated new functionality, such as supporting multiple operators, auditory communications, and expectations.

SITUATION AWARENESS MODEL VALIDATION: Three studies were undertaken to evaluate the validity of improvements made to MIDAS during 1998 and 1999 (MIDAS v1.0) with particular focus on the vision and SA models. The work was performed in collaboration with the Israeli Air Force under the auspices of a Memorandum of Agreement (17). Following a successful comparison of MIDAS predictions to the results of a laboratory study, a part-task simulation was completed using the Rotorcraft Part Task Laboratory Simulator. Pilots flew simulated missions in an AH-64 Longbow Apache designed to create situations predicted to generate high and low situation awareness. The mission of the co-pilot gunner was to hover and use a helmet mounted display (HMD) to view the battlefield and designate/identify objects with a computer keyboard. Visibility conditions could be good or bad; the local contrast of objects was either high or low; the number of non-target objects (clutter) was either high or low; and subjects may have been prepared (briefed) or not prepared (not-briefed) before each trial. Parallel MIDAS simulations produced results that were in the same direction as the human-in-the-loop results for visibility and briefing, but failed to simulate the effects of target contrast and clutter. Situation awareness results could not be compared due to differences in the measures used; MIDAS uses an analytical computation process whereas a SAGAT-type subjective rating was used for the piloted simulation.

SHUTTLE UPGRADE - At the request of the NASA Johnson Space Center, a model of the current cockpit of the Shuttle was developed with the intention of comparing the current configuration to that proposed for a planned upgrade. Most of the instrumentation in the shuttle is 1970s vintage. The caution and warning system is a particular problem, as it does not provide integrated failure information in a central location or succinct manner. The Shuttle Upgrade Program was initiated to develop an advanced orbiter cockpit with a human-computer interface designed to reduce workload through better displays and control designs, improve crew SA, increase flight crew autonomy and assist or automate complex procedures. MIDAS was used to create a virtual rendition of the cockpit and conduct a baseline nominal ascent simulation, providing a 
quantitative analysis of modifications in workload, SA and timing. Unfortunately, the project was not carried further due to funding cuts and concern that MIDAS v2.0 had not yet been subjected to a formal verification and validation process.

\section{MIDAS REDESIGN}

Redesign and re-implementation of much of the existing MIDAS system began in 1996. Over the many years of development and ad-hoc extensions, MIDAS v1.0 become very large and somewhat unwieldy from a software maintenance perspective, and contained much legacy code. The redesign had many goals, but the most significant included creating a cleanly designed system that combined the best of MIDAS v1.0 with fundamental enhancements to the human performance model, and a fully graphical user interface to enable "non-programmers", such as crewstation designers and cognitive modelers, to use MIDAS.

The Beta release of the new system was in 1999. The new system was built with object-oriented design and development methodology and supported multiple human operators, modeled audition, computed situation awareness, and allowed high level task abstraction. Most important, it had a graphical interface that eliminated the need for programming, with the notable exception of the process required to create operator procedures. For the first time since its inception, MIDAS models could be developed by people other than its programming staff. MIDAS v2.0, which runs on Silicon Graphics computers, has been steadily enhanced and applied to several modeling projects since its first release (it's still in Beta release mode). This section describes the current state of the system.

HUMAN OPERATOR MODEL - The human operator model is the central feature of MIDAS and simulates human behavior at both physical and cognitive levels. Its cognitive components include sensory input (visual and auditory), decision making, memory, attention, situation awareness, and output behavior (motor control). The relationships among these components are illustrated in Figure 3. The physical aspect of human behavior is simulated with anthropometric models, that provide

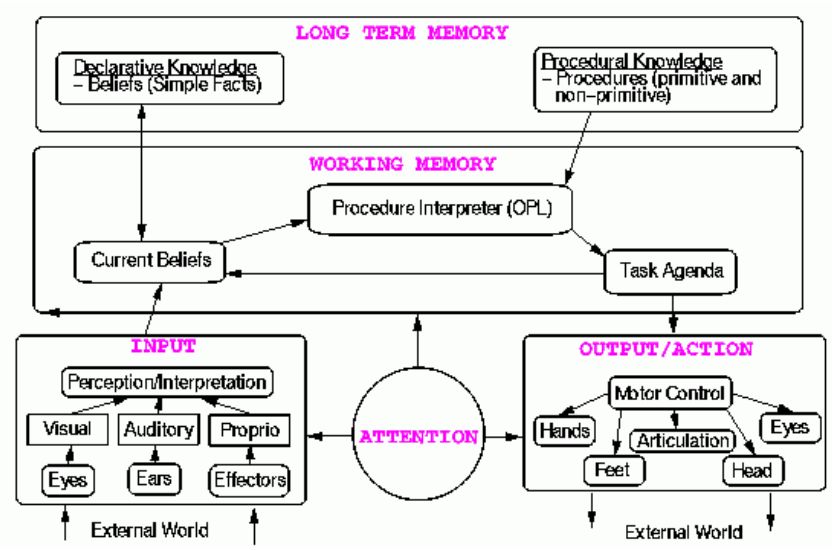

Figure 3: Human Operator Model structure animated views of the body. Because all of these models are described in detail in the MIDAS User's Manual (18) and human performance model overview (19), they will be summarized only briefly, with the exception of the decision-making model.

SENSORY INPUT - The operator obtains visual information about objects in the world from their symbolic representation (i.e., attributes that are attached to the objects) along with information about the objects' surroundings such as ambient lighting. The vision model differentiates between peripheral and foveal vision. While foveal vision involves fixation and attention on a specific object, peripheral vision allows the modeling of significant visual distractions that may divert the operator's attention temporarily or indefinitely. MIDAS v2.0 conceptually differentiates interior (within the crew station) and exterior (outside the crew station) vision. For interior vision, the operator is assumed to have a mental representation of equipment he interacts with including its location and function. In contrast, an operator must recognize or identify an exterior object before he can reason about it. Perception of exterior vision takes place in three stages: detection, recognition, and identification, and the perception level attained is dependant on various factors defined in the MIDAS perceivability model, a component of the vision model.

Auditory perception occurs only within the crew station (hearing of exterior sounds is not yet supported, unless it is channeled through equipment contained within the crew station representation, such as a speaker). Auditory signals and speech messages are perceived in two stages, detection and comprehension, and cannot be partially comprehended - if a listening task is interrupted the entire content will be lost.

DECISION MAKING - In sharp contrast to MIDAS v1.0, which required specification of all of an operators activities at the primitive task level, human behavior is now specified in a much more abstract way using a highlevel scripting language called the Operator Procedure Language, or OPL, which serves as the front end to a reactive planning system $(20,21)$. As its name implies, the central construct of this language is the procedure, which represents an atomic unit of the operator's knowledge. Procedures can be thought of as instructions for accomplishing a task, much as a procedure in a programming language (technically, OPL is a programming language), OPL procedures can take input (arguments) and invoke (call) other procedures. Such idioms fit naturally into human procedure modeling, as illustrated by the following example of OPL.

Table 1: Procedural representation of turning an ignition key

(procedure (turn-ignition)

(task (move-right-hand ignition))

(task (turn-clockwise-with-right-hand ignition))) 
(procedure (move-right-hand obj)

(task (move-effector-primitive right-hand obj)))

(procedure (turn-clockwise-with-right-hand obj) (task (turn-object-primitive right-hand obj clockwise)))

The procedures listed in Table 1 model the turning of an ignition key. The first procedure might be scenariospecific and hence written by the MIDAS user. The latter two exemplify library procedures known by simulated MIDAS operators. They also call primitive procedures (e.g., move-effector-primitive, turn-object-primitive). A primitive procedure specifies a basic task that is not decomposed into other OPL procedures but rather executed directly by an action (e.g. a physical task such as reaching) or in memory (e.g. remembering a fact or performing a computation). This example illustrates only simple, sequential behavior and primitive action, but OPL includes constructs for modeling more complex activities, such as selecting between alternatives, repetition, waiting (e.g., passively monitoring for a perceived condition), and concurrent tasks.

MEMORY - The current memory model in MIDAS v2.0 is simple. It is essentially a database of assertions, or beliefs. A belief is represented as a symbolic expression that usually denotes the property of an object, and is illustrated by the examples in Table 2. Memory can be examined in powerful ways by means of a querying language built into OPL. For example, one can easily write a procedure for reporting to headquarters about all instances of blue vehicles seen in a given area.

Table 2: Examples of memory representations
(color car1 blue)
(raining? false)
(wipers-on? false)
(aircraft (style rotorcraft) (location (waypoint 3)))

ATTENTION - The MIDAS attention model, is based on Wicken's Multiple Resource Theory (9). It acts as a mediator that maintains an account of attentional resources in six different "channels". Two channels pertain to encoding (visual and auditory input), two to cognitive processing (spatial and verbal) and the remaining two to responding (motor and voice output). Before a primitive task is initiated, the necessary attentional resources must be secured from the model. If sufficient resources are not available, the task performance may be degraded. The load on each channel is computed using a matrix of resource coefficients, which were estimated using Multiple Resource Theory.

SITUATION AWARENESS - The SA model is based on a computational representation developed by Jay Shively (6). It computes two quantities: actual $S A$ and perceived $S A$. The actual SA of an operator is defined as the portion of situational elements that he knows relative to the situation elements that he would know in the ideal state. Perceived SA differs from Actual SA only in that it does not include elements for which the operator has no knowledge. Situational elements can be either specific objects in the crew station or environment that define a "situation" or operationally pertinent information in the operator's memory.

OUTPUT BEHAVIOR - Output behavior is regulated by a motor control process. If required resources are available, a motor activity that corresponds to a primitive procedure is created. Both the operator's physical actions and their effects on equipment and/or environment objects are modeled. Activities such as manipulating equipment, fixating on an object, or making a speech utterance are all supported as primitive motor outputs. There are about 30 such primitive tasks available in the Procedure Library. Each task has load values defined for each of the six resource channels (10).

ANTHROPOMETRIC MODEL - Used primarily for visualization, the anthropometric model provides a 3D animated graphical representation of the modeled human operator. There are two anthropometric models available in MIDAS. Jack $^{\circledR}$, a product of Unigraphics Solutions, Inc., is a full body figure with advanced capabilities and very realistic movement (7). Because the use of Jack $^{\circledR}$ requires a runtime license, a simpler anthropometric model, consisting of just a head and hands, is included with MIDAS v2.0. From a modeling perspective, both are equivalent in terms of the simulation results that will be produced.

CREWSTATION/EQUIPMENT MODEL- A crew station, such as an aircraft cockpit or nuclear power plant control room, is the essential component of a MIDAS simulation. "Crew station" defines a collection of equipment components with which operators can interact. There are several kinds of equipment component models: discrete-state components and continuous components. Discrete-state components are used to model equipment whose behavior is characterized by a finite number of distinct states, such as toggle switches. A discrete-state component is a finite state machine, in essence, with the added ability to send messages to other components upon state changes. Continuous components are used to represent equipment that has a continuous range of values, such as a pressure meter or compass needle. There are several varieties of continuous components, each also having the ability to send messages upon value change. This message-sending capability allows the definition of fairly complex equipment components and assemblies, including approximations of multifunction displays. Crew stations and equipment may be given graphical representation for animation by assigning geometry to the models. MIDAS supports multiple crewstations per vehicle and multiple operators per crewstation. The crewstation model supports cognitive modeling by allowing components and component states to be assigned visual or auditory attributes that are processed by the perception model. 
VEHICLE MODEL - MIDAS v2.0 provides the ability to model moving vehicles. A modeled vehicle in MIDAS is the combination of a guidance/dynamics model and a visual representation. The guidance/dynamics model moves the vehicle along a prescribed route in a particular fashion. MIDAS provides two guidance/dynamics models: a nap-of-the-earth (NoE) helicopter model developed at NASA Ames $(22,23)$ and a simple point mass model. The latter is used to model movement of arbitrary vehicles in a generic way. The visual representation of a vehicle is CAD geometry, chosen from the MIDAS geometry library or provided by the modeler. A MIDAS simulation may contain any number of vehicles.

ENVIRONMENT MODEL - MIDAS v2.0 provides tools to model the environment outside of a vehicle (or outside of a crew station if it is not in a vehicle) and components for creating "outdoor" settings that may include terrain, specific features, and weather. Non-terrestrial environments, such as air and space, may be represented by this model, as well if appropriate geometry for features is available. Terrain is modeled as a single object representing a rectangular plot of ground. Features are simple objects of interests such as trees, buildings, and non-moving vehicles that do not have any inherent behavior. A single weather condition may be applied to the environment by specifying lighting and visibility values. These are used by the visual perception model. Perception of the environment is made possible by assigning visual attributes to environmental objects. The capability of "hearing" noises in the environment is not yet supported.

\section{SIMULATION SYSTEM}

The MIDAS simulation infrastructure consists of an engine/executive, data collection and event generation mechanisms, and a framework called the provisioning system that supports dynamic simulation modification. The simulation executive uses a discrete-event, fixedtime increment approach for advancing the simulation. A global simulation clock is advanced by a fixed time unit at each iteration (one unit $=100 \mathrm{~ms}$ ). Every entity in the simulation performs $100 \mathrm{~ms}$ worth of activity in a sequenced fashion. This is a very simple and fairly inefficient approach to simulation in general, but serves well the MIDAS' modeling needs and provides an easy way to control time compression and coordinate animations.

The data collection system provides the framework for generating runtime data that is graphically displayed while the simulation runs and saved in files for post-run analysis. The event mechanism provides a way to make timed events occur while the simulation runs. Events are either converted into object attributes or messages sent to equipment components. Their timing may have stochastic variance. The provisioning system is not directly visible to the user, however, it allows the user to make changes to simulation models or parameters and then re-runs the simulation, without having to restart
MIDAS or reload the simulation. This is in sharp contrast to the MIDAS v1.0, that required re-compiling of source code that had been edited to modify a simulation.

\section{USER INTERFACE}

One of the major strengths of MIDAS v2.0 is its user interface. It provides an interactive graphical environment for creating models, specifying simulations, running simulations, and viewing analysis data. The user interface is organized into a hierarchical system of screens or editors that can be navigated quickly using a tabbed file deck metaphor (see Figure 4). Many different views of the simulation are provided. Structure Views represent the environment, crew station, and scenario as graphs containing editable nodes. The Geometry View shows the corresponding $3 \mathrm{D}$ visualization and allows direct manipulation of the geometry (e.g. placement, rotation). The Outline View gives a textual listing of defined components and provides a search feature. When the simulation is running, Animation Viewers show the model in action and can be assigned various camera positions such as a wingman's view, "Bird's eye" view, and an operator's eye view. Data displays show updating views of attention demands (workload), SA, and other information. Finally, post-run analysis data is summarized in graphs that contain basic statistics computed on workload and SA values.

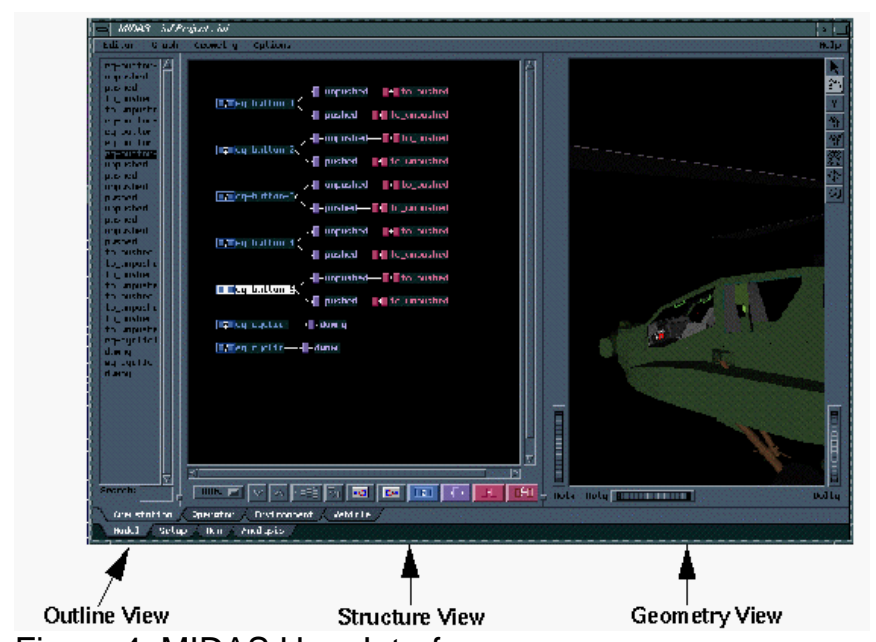

Figure 4: MIDAS User Interface

\section{VALIDATION OF MIDAS V2.0}

The most recent application of MIDAS has been a simulation of a man-in-the loop experiment. The goal was to verify the validity of MIDAS v2.0 by comparing MIDAS results to those obtained in a piloted, high-fidelity rotorcraft simulation. Micro Analysis and Design Inc (MA\&D) and the US Army developed a MIDAS simulation of the Simulation Awareness Model Simulation (SAMSIM) conducted at Ames in the Vertical Motion Simulator (VMS) in March-April 2000 under the direction of Jay Shively. In MIDAS, an attempt was made to simulate the conditions of the man-in-the-loop simulator and determine whether simulated target detections and pilot SA corresponded to the results of the VMS experiment. 
Pilot's were instructed to fly a designated NoE course at a prescribed altitude and airspeed (100 ft at 80 knots). They were told to report all ground elements (friend or foe) by radio, indicating approximate map locations by stating the bearing and distance to the target. They were also told to report all aircraft sightings and equipment failures. The modeled scenario had 14 targets ( 9 tanks and 5 vans) distributed on a $5 \mathrm{~km} \times 9 \mathrm{~km}$ terrain representation. Pilot's were told to report a bearing to the target from their current location and its estimated distance off the flight path (each target was either $500 \mathrm{ft}$ or $1000 \mathrm{ft}$ off the flight path). Figure 5 represents the simulated flight path and target locations.

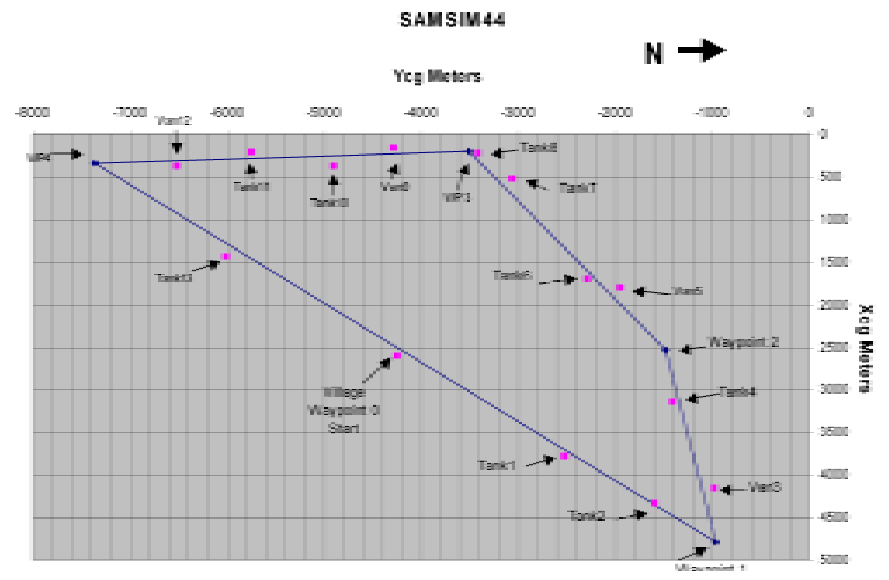

Figure 5 Flight Path

High fidelity representations of the VMS simulator used to conduct the experiment were implemented using MIDAS. Figures 6 and 7 show a picture of the actual crew station versus the modeled crew station.

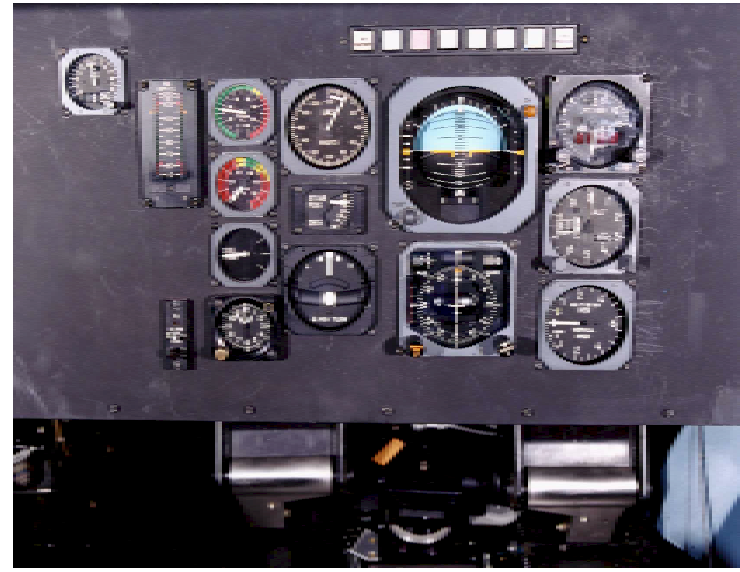

Figure 6: Photo of VMS simulator instruments

Using OPL, we have constructed Pilot behaviors to search, detect, identify and report visible targets. Remember instrument settings, and identify if an instrument stops correctly functioning.

One limiting factor encountered with the simulation environment is that all operator inputs are either declarative knowledge or must come through the auditory and visual perceptual models in MIDAS. These limits can be somewhat mitigated through the scenario editor where time based events can be injected into the simulated stream of events.

METRICS - SAMSIM was not conducted with the MIDAS representation in mind. Thus, the metrics used during the man-in-the-loop experiment do not provide measures that are directly comparable to those generated by MIDAS v20. The experimental measures used were subjective measures of SA, using the Situation Awareness Rating Technique (SART), and workload, using the NASA-Task Load Index (NASA-TLX)) collected after the completion of a mission. An approach using interruptions in the Pilot's flight profile, with intermediate data collections, similar to that used in the Situation Awareness Global Assessment Technique (SAGAT) may have provided a better measure for comparison with the objective SAMSIM and workload measured from MIDAS. We will attempt to integrate the simulated SAMSIM and W/Index results over time and compare them with the empirical SART and TLX values collected from the experiment.

\section{SIMULATION DEVELOPMENT STATUS}

The MIDAS simulation is still a work in progress. We are currently addressing three confounding problems. The first is that the vision model sees through the terrain; thus, the operator can perceive the same target multiple times. We are working on a method to solve this problem and then calibrate the correct vision model used within MIDAS (light fog or hazy day). There was no measure of the environmental conditions in SAMSIM that directly correlate with the environmental conditions simulated in MIDAS. Finally, the NoE flight model has not yet been re-

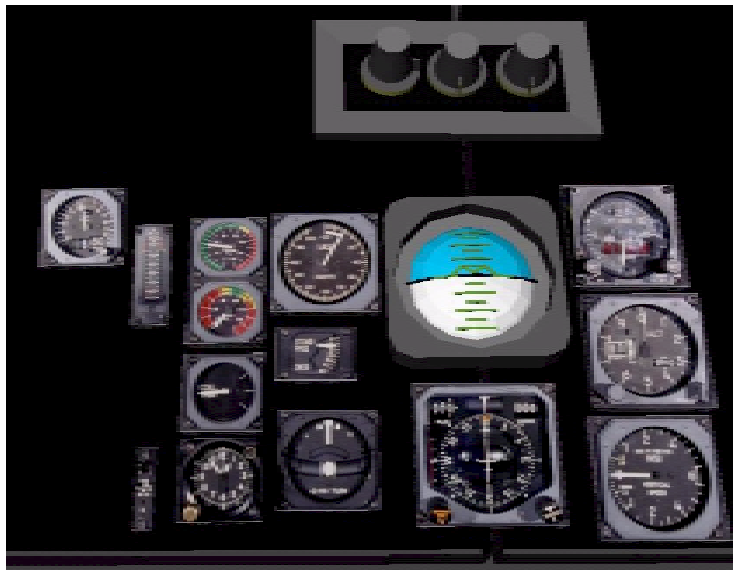

Figure 7: MIDAS version of instrument panel

integrated into MIDAS v2.0 Beta, thus we used a simple navigation model and defined multiple waypoints to emulate the flight profile used in the experiment. This changing profile affects the setting of the proper SA context for different legs of the mission.

Once completed, we will be able to extract a timeline of workload and situational awareness, as well as a high fidelity visualization of pilot activities while conducting the mission, similar to that shown in Figure 8. 


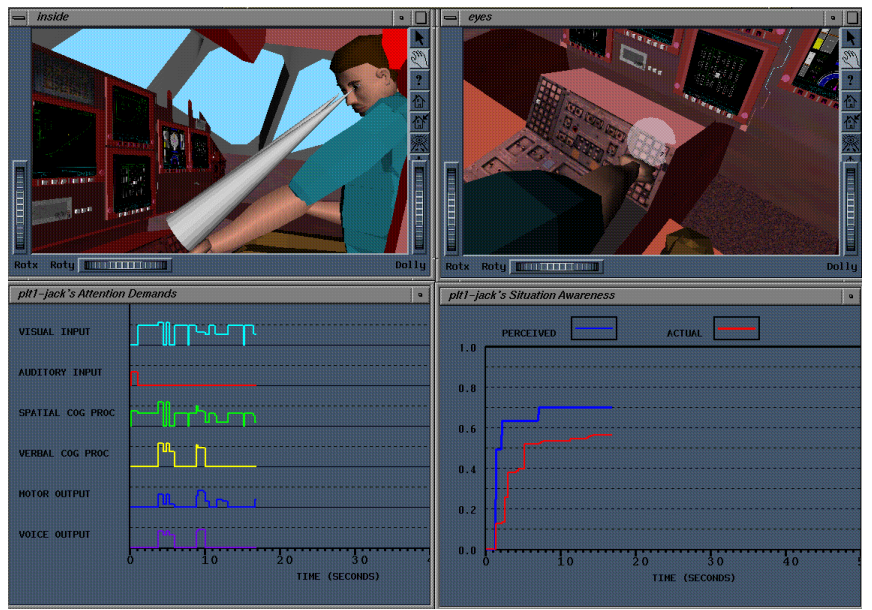

Figure 8: MIDAS simulation run (courtesy of San Jose State University Human Automation Integration Laboratory)

\section{CONCLUSIONS}

MIDAS gives users the ability to model the functional and physical aspects of the operator, the system, and the environment, and to bring these models together in an interactive, event-filled simulation for quantitative and visual analysis. Operator behavior within a MIDAS simulation is driven by a set of user inputs specifying operator goals, procedures for achieving those goals, and declarative knowledge appropriate to a given simulation. These asserted knowledge structures interact with and are moderated by embedded models of perception for extracting information from the modeled world and embedded models of cognition for managing resources, memory, and actions. In this way, MIDAS seeks to capture the perceptual-cognitive cycle of real world operators who work towards their most immediate goals given their perception of the situation and within the limitations of their physical and cognitive capacities. In MIDAS, as in the real world, perceived changes in the world - new information or events - may cause changes in the adjudged context of the situation triggering new goals or altering methods to achieve current goals. Such perceived changes may be precipitated through the behavior of other modeled entities or by user specified time-based, condition-based, or probabilistic events set within the simulation (e.g., a system failure or the receipt of an incoming air traffic control clearance). It may, in fact, be the impact of the operator's own actions which lead to changes in context and goals.

This complex interplay between top-down and bottom-up processes makes possible the emergence of unforeseen, unpredicted behaviors. Task time-lines are not an input requirement of a MIDAS simulation, but rather one of its outputs. Behavior flows from and is sensitive to the goals, knowledge, and domain expertise imparted to the virtual operator, the perceptual availability of information in the constructed environment, and the nature and timing of external events

MIDAS has proven to be a flexible and useful tool for representing humans operating in a variety of environments. Its greatest strength lies in its ability to represent conceptual designs or candidate procedures in software and then allow a designer or potential user see them operated by a virtual crew members in the context of a simulation of the target environment. The recent addition of a graphical user interface has made MIDAS accessible to a broader range of potential users. The government has done what it set out to do; spur development of state-of-the-art human performance modeling tools that integrate relevant psychological models of cognition, perception, memory and so on into a design environment that includes human factors as an integral component. As the user community for MIDAS v2.0 increases, the hope is that ever more capable models will be incorporated allowing MIDAS to achieve its full potential.

\section{REFERENCES}

1 Hartzell, E. J. (1990) Army-NASA Aircrew/Aircraft Integration Program $\left(A^{3} I\right)$.. Executive Summary. Moffett Field, CA: NASA-Ames Research Center.

2 Smith, B.R. \& Tyler, S.W. (1997) The design and application of MIDAS: A constructive simulation for human-system analysis. $2^{\text {nd }}$ Simulation Technology \& Training Conference (SIMTECT), Canberra, Australia 3 Elkind, J. I., Card, S., Hockberg, J., \& Huey, B. M. (Eds.) (1989) Human Performance Models for Computer-Aided Engineering. Washington, CD: National Academy Press.

4 R. Shankar, (1991) Z-Scheduler: Integrating Theories of Scheduling Behavior into a Computational Model. In Proceedings of the IEEE Conference on Systems, Man, and Cybernetics, 1219-1223.

5 Remington, R. W., Johnston, J. C., \& Yantis, S. (1992) Involuntary Attentional Capture by Abrubt Onsets. Perception \& Psychophysics, 51 (3), 279-290.

6 Shively, R.J., Brickner, M. \& Silbiger, J. (1997) A Computational Model of Situational Awareness Instantiated in MIDAS. In Proceedings of the Ninth International Symposium on Aviation Psychology. Columbus, Ohio: University of Ohio, 1454-1459.

7 Badler, C.B. Phillips, \& B.L. Webber. (1993) Simulating Humans: Computer Graphics, Anima-tion, and Control. Oxford University Press, 1993

8 Lubin, J. (1995) A visual Discrimination Model for Imaging System Design and evaluation, David Sarnoff Research center.

9 Wickens, C. D. (1980) The structure of attentional resources. In R. Nickerson (Ed.), Attention and performance VIII Hillsdale, NJ: Lawrence Erlbaum, 239257.

10 Aldrich, T. B., Szabo, S. M., \& Bierbaum, C. R. (1989) The Development and Application of Models to Predict Operator Workload During System Design". In G. MacMillan, D. Beevis, E. Salas, M. Strub, R. Sutton \& L. Van Breda (Eds.), Applications of Human Performance Models to System Design._New York: Plenum Press, 6580. 
11 Rasmussen, J. (1983) Skills, Rules, and Knowledge; Signals, Signs and Symbols, and Other Distinctions in Human Performance Models. IEEE Transactions on Systems, Man, and Cybernetics, Vol. SMC-13, No. 3, 257-266. Baddeley, A. D. (1986) Working Memory. Oxford University Press.

12 Hoecker, D. G., Roth, E. M., Corker, K. M., Lipner, M. H., \& Bunzol, M. S. (1994) Man-machine Design and Analysis System (MIDAS) Applied to a Computer-Based Procedure-Aiding System. In Proceeding of the 38th Annual Meeting of the Human Factors and Ergonomics Society. Santa Monica: Human Factors and Ergonomics Society.

13 Atencio, A., Jr., Shively, R. J. \& and Shankar, R. (1996) Evaluation of Air Warrior Baselines in a Longbow Apache Helicopter Crewstation in a MIDAS Simulation, In Proceedings of the American helicopter Society 52nd Annual Forum, Washington, D.C

14 Atencio, A., Jr., Banda, C., and Tamasi, G. (1998) Evaluation of a Short Haul Civil Tiltrotor $\mathrm{SH}(\mathrm{CT})$ in Emergency Go-Around - A MIDAS Simulation, In Proceedings of the American Helicopter Society 54th Annual Forum. Washington, DC.

15 Corker, K. M. \& Pisanich, G. (1995) Analysis and Modeling of Flight Crew Performance in Automated Air Traffic Management Systems. In Proceedings of the 6th IFAC/IFIP/IFORS/IEA Symposium: Analysis, Design, and Evaluation of Man-Machine Systems, Boston, MA, 1995

16 Corker, K. M., Lozito, S., \& Pisanich, G. (1995) Flight Crew Performance in Automated Air Traffic Management. In Fuller, Johnston, \& McDonald (Eds.) Human Factors in Aviation Operation (Vol. 3), Hants, UK: Avebury Aviation.

17 Shively, R. J., Burdick, M. D., Brickner, M., Nadler, I., \& Silbiger, J. (2000) Comparison of a manned helicopter simulation to a computer-based human performance model. Proceedings of the American Helicopter Society 56th Annual Forum, Virginia Beach, Virginia.

18 Atencio, A., Dalal, K. M., Goodman, A., Neukom, C., Pearl, C. (1999-2001) Midas User Manual. MIDAS web site.

19 Tyler, S. W., Neukom, C., Logan, M., Shively, J (1998). The MIDAS Human Performance Model Presented at the Human Factors and Ergonomics Society, 42nd Annual Meeting, 1998.

20 Firby, R. J. (1989) Adaptive Execution in Complex Dynamic World. PhD Thesis. New haven, CN: Yale University.

21 Freed, M. A. (1998) Simulating Human Performance in Complex, Dynamic Environments. PhD Thesis, Northwestern University

22 Lewis, M.S. and Aiken, E.W., (1985) Piloted Simulation of One-on-One helicopter Air Combat at NOE Flight Level (Appendix) (NASA TM 86686) Washington, DC: National Aeronautics and Space Administration.

23 Phatak, Anil V. and Tran, Hien T. (1987) $A^{3}$ I Autopilot/Guidance Program -Version TDRIVE2. (AMA Report 252-1) Mountain View, CA: Analytical Mechanics Associates. 